\title{
RETROCOLLIS, ANTEROCOLLIS OR HEAD TREMOR MAY PREDICT THE SPREADING OF DYSTONIC MOVEMENTS IN PRIMARY CERVICAL DYSTONIA
}

\author{
Clecio Godeiro-Junior, Andre Carvalho Felício, Patrícia Maria de Carvalho Aguiar, \\ Vanderci Borges, Sonia Maria Azevedo Silva, Henrique Ballalai Ferraz
}

\begin{abstract}
Background and Purpose: Few studies have attempted to develop clinical predictors for cervical dystonia (CD) aiming at progression of the dystonic movement. Method: We retrospectively evaluated 73 patients with primary $C D$ who underwent treatment with Botulinum toxin type-A (BTX-A). The patients were assembled in two groups according to the spread of dystonia during follow-up: spreading and non-spreading CD. We performed a binary logistic regression model using spreading of cervical dystonia as dependent variable aiming to find covariates which increase the risk of spreading. Results: Our logistic regression model found the following covariates and their respective risk ratios: time of disease $>18.5$ months $=2.4$, retrocollis $=1.9$, anterocollis=1.8, head tremor $=1.6$. Conclusion: Time of disease $>18.5$ months, retrocollis, anterocollis and head tremor may predict spreading of dystonic movement to other regions of the body in CD patients.
\end{abstract}

KEY WORDS: cervical dystonia, progression, retrocollis, anterocollis, head tremor.

\begin{abstract}
Retrocolis, anterocolis ou tremor cefálico podem predizer a progressão dos movimentos distônicos em pacientes com distonia cervical primária

Resumo - Introdução: Poucos estudos avaliam preditores clínicos de progressão dos movimentos distônicos, para além da região cervical, em pacientes com distonia cervical (DC) primária. Método: Avaliamos, retrospectivamente, 73 pacientes com DC primária, que tinham sido submetidos ao tratamento com a toxina botulínica tipo $\mathrm{A}$ (BTX-A). Estes pacientes foram divididos em dois grupos de acordo com a progressão ou não da DC para outras áreas do corpo. Aplicamos um modelo de regressão logística binária usando a progressão da distonia como variável dependente com o objetivo de identificar co-variáveis que aumentassem o risco de progressão. Resultados: $\mathrm{O}$ modelo de regressão logístico identificou as seguintes co-variáveis como preditoras de progressão e seus respectivos índices de risco: tempo de doença $>18,5$ meses $=2,4$, retrocolis=1,9, anterocolis=1,8, tremor cefálico=1,6. Conclusão: Tempo de doença $>18,5$ meses, retrocolis, anterocolis, e tremor cefálico podem predizer a progressão do movimento distônico para outras regiões do corpo em pacientes com DC primária.
\end{abstract}

PALAVRAS-CHAVE: distonia cervical, progressão, retrocolis, anterocolis, tremor cefálico.

Clinical studies of dystonia have recognized a relationship between the age at symptom onset, affected body region at onset, and disease progression ${ }^{1,2}$. The natural history of cervical dystonia (CD) is not clear. The majority of patients deteriorates during the first five years, become static for the next 5 years, and then tend to show slight improvement ${ }^{3}$. Therefore, an important concern is the spread of CD to other body parts. Previous studies that evaluated clinical and demographic characteristics of patients with $C D$ were not able to demonstrate predictable variables for the risk of $C D$ progression ${ }^{4-8}$.

The Tsui scale score has been the most widely used instrument developed to follow-up patients with $C D$ who undergo treatment with Botulinum toxin type-A (BTX-A). The parameters evaluated through this scale are amplitude and duration of sustained movements, shoulder el-

Movement Disorders Unit, Department of Neurology and Neurosurgery, Federal University of São Paulo, São Paulo SP, Brazil.

Received 27 October 2008, received in final form 23 January 2009. Accepted 18 April 2009.

Dr. Clecio Godeiro-Junior - Rua Monsenhor José Paulino 1061 / 101 - 59022-200 Natal RN - Brasil. E-mail: cleciojunior@yahoo.com.br 
evation, pain and head tremor. The score ranges from 0 to $25^{9}$. Unfortunately, this instrument can not be used to predict the natural history of $C D$.

The aim of our study was to find clinical and epidemiological predictors for $C D$ to spread to other parts of the body.

\section{METHOD}

We retrospectively analyzed 184 charts of patients with CD who underwent medical treatment with BTX-A at the Movement Disorders Unit of our institution between 1990 and 2007.

We defined $C D$ as primary, when there was no evidence on history, clinical and neurological examination or laboratory investigation of any identifiable cause of the dystonia; and secondary, when the cause of dystonia was identifiable such as anoxia, central (brain and spinal cord) or peripheral trauma, peripheral neuropathy, infection, metabolic disorder, previous use of neuroleptic drugs or when associated to other neurodegenerative disease such as atypical parkinsonism or Huntington's disease.

We identified 73 patients with primary CD and reviewed their medical chart for various demographic and clinical characteristics. We classified the patients according to gender, age of onset, family history of $C D$ and Tsui scale score at first medical evaluation. Details of the dystonic movement at the first medical evaluation were reviewed: torticollis, anterocollis, retrocollis, laterocollis, head tremor and pain. We considered only dystonic head tremor with positive antagonistic geste and not overlapping essential head tremor.

The patients were divided according to the spread of dystonia during follow-up into two groups: spreading and non-spreading CD. We considered CD spreading when the dystonic movement extended to a different body region outside the cervical region. The pattern of progression included the following dystonic subtypes: blepharospasm, Meige syndrome, lower or upper limb dystonia, hemidystonia, multifocal or generalized dystonia.

Statistical analysis for continuous variables and comparisons between subgroups were performed by Student t-test (or MannWhitney test, for non-parametric data); for categorical variables, comparisons between groups were performed using the Chisquare test. Significant values of $p$ were set at $\leq 0.05$. The statistical analyses were performed using software Prism 3.0. Parametric data is presented as mean \pm standard deviation (SD) and nonparametric data as median \pm percentile $\left(25^{\text {th }}\right.$ and $75^{\text {th }}$ percentiles).

We also performed a binary logistic regression model using spreading of cervical dystonia as dependent variable and the following variables were candidates for covariates: familial history, onset age $<28$ years, time of disease $>18.5$ months, score Tsui $>7.5$, head tremor, pain, rotation, laterocollis, retrocollis and anterocollis. Time of disease is defined as the period between the first clinical presentation and the first clinical evaluation in our department. We chose onset-age $<28$ years as a candidate variable because it defines an early onset dystonia and patients in this category present a higher risk of spreading, ${ }^{9,10}$. We transformed continuous variables (time of disease and Tsui score) into categorical ones (time of disease >18.5 months and score Tsui >7.5) appling ROC (receiver operating characteristic) curve method to our own data: the value with highest specificity and sensitivity for each variable was used as cut-off in the categorization process. To select the covariates we used likelihood ratio with value of significance of $p<0.2$. Variables identified as covariates $(p<0.2)$ were included in a binary logistic regression model. These statistical analyses were performed using software SPSS 13.0 for Windows.

This protocol was submitted and approved by the Ethical Committee of our institution.

Table 1. Demographic and clinical data of patients with spreading and non-spreading CD.

\begin{tabular}{lccc}
\hline & Spreading & Non-Spreading & p value \\
\hline Population sample with CD n=73 & $25(34.3 \%)$ & $48(65.7 \%)$ & \\
Male (M) & $8(32 \%)$ & $23(47.9 \%)$ & \\
Female (F) & $17(68 \%)$ & $25(52.1 \%)$ & \\
F:M & $2.2: 1$ & $1.1: 1$ & $0.2^{\mathrm{a}}$ \\
Age at onset (years) & $45.7 \pm 3.6^{*}$ & $37.1 \pm 1.9^{*}$ & $0.02^{\mathrm{b}}$ \\
Disease duration (months) & $48(36-120)^{\#}$ & $48(12-90)^{\#}$ & $0.1^{\mathrm{c}}$ \\
Follow-up (months) & $38(14.5-57.5)^{\#}$ & $43(15.5-71)^{\#}$ & $0.4^{\mathrm{c}}$ \\
Point of spreading (months) & $24(12-38)^{\#}$ & - & - \\
Family history of CD & $2(8 \%)$ & $4(8.34 \%)$ & $0.69^{\mathrm{a}}$ \\
Tsui scale score & $10(8-13)^{\#}$ & $8.5(6-10)^{\#}$ & $0.03^{\mathrm{c}}$ \\
Head tremor & $7(28 \%)$ & $23(48 \%)$ & $0.2^{\mathrm{a}}$ \\
Pain & $19(76 \%)$ & $35(73 \%)$ & $0.9^{\mathrm{a}}$ \\
Torticollis & $41(89.2 \%)$ & $69(95.8 \%)$ & $0.3^{\mathrm{a}}$ \\
Laterocollis & $21(45.6 \%)$ & $35(48.6 \%)$ & $0.9^{\mathrm{a}}$ \\
Anterocollis & $12(26.1 \%)$ & $9(12.5 \%)$ & $0.1^{\mathrm{a}}$ \\
Retrocollis & $21(45.6 \%)$ & $23(32 \%)$ & $0.13^{\mathrm{a}}$ \\
\hline
\end{tabular}

*Data are presented as mean \pm SD; ${ }^{\sharp}$ median and percentile; ${ }^{a}$ chi-square test; ${ }^{b}$ Student $t$-test; ${ }^{\mathrm{c}}$ Mann- Whitney test. 
Table 2. Phenomenology of cervical dystonia movement in both groups.

\begin{tabular}{lcc}
\hline Phenomenology & Spreading & $\begin{array}{c}\text { Non- } \\
\text { Spreading }\end{array}$ \\
\hline Rotation & $3(12 \%)$ & $14(29.2 \%)$ \\
Anterocollis & $1(4 \%)$ & 0 \\
Retrocollis & 0 & 0 \\
Laterocollis & 0 & 0 \\
Rotation + Laterocollis & $2(8 \%)$ & $14(29.2 \%)$ \\
Rotation + Anterocollis & $5(20 \%)$ & $3(6.2 \%)$ \\
Rotation + Retrocollis & $8(32 \%)$ & $8(16.7 \%)$ \\
Retrocollis + Latercollis & $1(4 \%)$ & $1(2.1 \%)$ \\
Rotation + Latercollis + Anterocollis & $1(4 \%)$ & $4(8.3 \%)$ \\
Rotation + Latercollis + Retrocollis & $4(16 \%)$ & $4(8.3 \%)$ \\
\hline
\end{tabular}

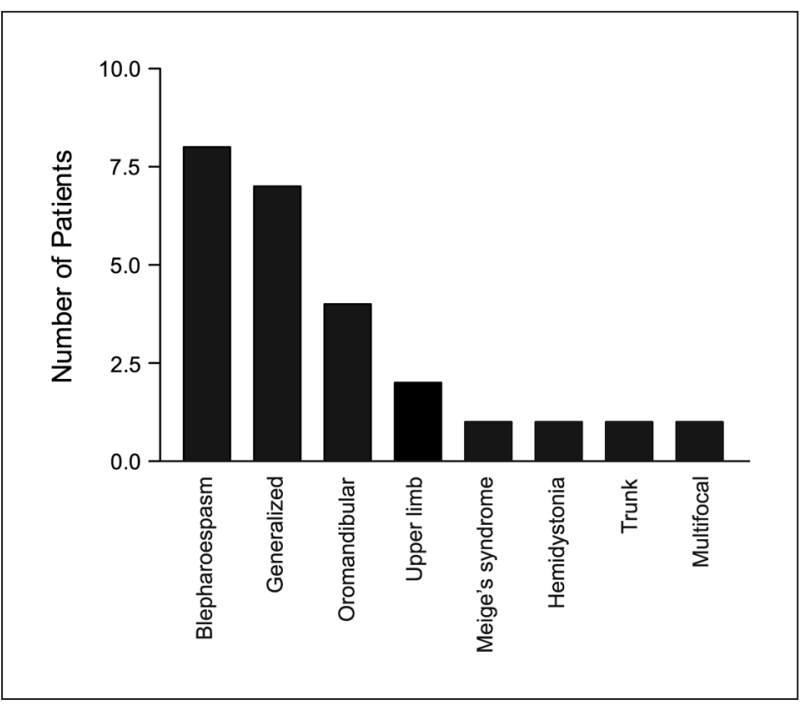

Figure. Pattern of spreading in patients wit primary $C D$.

Table 3. Candidate variables for logistic regression model.

\begin{tabular}{lcccc}
\hline Candidate variable & Odds ratio & $\begin{array}{c}95 \% \text { Confidence } \\
\text { interval (C.I.) }\end{array}$ & Likelihood ratio & p value \\
\hline Onset age $<28$ years & 1.2 & $0.38-3.8$ & 0.09 & 0.7 \\
Family history & 0.95 & $0.16-5.6$ & 0.02 & 0.9 \\
Time of disease $>18.5$ months & 7.5 & $1.6-35.7$ & 9.22 & 0.002 \\
Tsui score $>7.5$ & 3.75 & $1.1-12.6$ & 3.81 & 0.02 \\
Head tremor & 0.43 & $0.15-1.2$ & 2.7 & 0.09 \\
Pain & 1.2 & $0.38-3.6$ & 0.08 & 0.77 \\
Rotation & 1.1 & $0.96-1.2$ & 2.17 & 0.14 \\
Laterocollis & 0.6 & $0.2-1.65$ & 0.96 & 0.33 \\
Retrocollis & 2.77 & $0.99-7.68$ & 3.8 & 0.05 \\
Anterocollis & 2.7 & $0.8-9.3$ & 2.6 & 0.11 \\
\hline
\end{tabular}

Table 4. Spreading risk ratio of the variables after binary logistic regression.

\begin{tabular}{lccc}
\hline & Spreading risk ratio & Interval of confidence $95 \%$ & $p$ value \\
\hline Time of disease $>18.5$ months & 2.4 & $0.02-0.54$ & 0.008 \\
Retrocollis & 1.9 & $0.04-0-0.57$ & 0.006 \\
Anterocollis & 1.8 & $0.04-0.85$ & 0.031 \\
Head tremor & 1.6 & $1.4-18.5$ & 0.014 \\
\hline
\end{tabular}

\section{RESULTS}

In 25 patients (34.3\%), dystonia spreaded from the cervical region to other body regions. The occurrence of disease spreading presented a median of $24\left(12-38,25^{\text {th }}\right.$ and $75^{\text {th }}$ percentiles) months. Clinical and demographic data of both groups are presented in Table 1.

In the spreading group most of patients presented rotation associated to anterocollis, retrocollis or laterocollis. In the non-spreading group, rotation or rotation associ- ated with laterocollis. The phenomenology of CD in both groups is presented in Table 2.

The pattern of dystonia spreading was: blepharospasm in 8 (32\%); generalized in 7 (28\%); oromandibular in 4 (16\%); upper limb in 2 (8\%); Meige's syndrome in 1(4\%); hemidystonia in 1 (4\%); trunk in 1(4\%); multifocal in $1(4 \%)$. We did not observe isolated spreading to the lower limb. Fig 1 shows the pattern of spreading found.

In our model of logistic regression, the variable elect- 
ed as covariates: time of disease $>18.5$ months, score Tsui $>7.5$ at first clinical evaluation, rotation, retrocollis, anterocollis and head tremor (Table 3). These variables were included as covariates in a binary logistic regression model using spreading as dependent variable. Table 4 shows the variables identified as predictors of spreading and their respective risk ratio and confidence intervals.

\section{DISCUSSION}

Among 73 patients with primary CD, 34.3\% $(n=25)$ had progression beyond the neck region, and our model of logistic regression identified predictors of spreading: time of disease $>18.5$ months, retrocollis, anterocollis, and head tremor.

In previous reports, the clinical characteristics of CD such as head tremor, pain and direction of the movement (rotation, laterocollis, anterocollis and/or retrocollis) have been suggested as predictors of remission, but not of progression ${ }^{4,5}$. In some reports, those patients without head tremor, without pain and with a simple form of CD had more chances of remission ${ }^{5,11}$. Our data suggest that in our group of patients, the presence of anterocollis (risk ratio=1.8), retrocollis (risk ratio=1.9) or head tremor (risk ratio=1.6) predicts a higher chance of spreading. Interestingly, there is no significant difference in the percentage of patients with these characteristics between spreading and non-spreading groups in their first medical evaluation (Table 1). Tsui score is even higher in the spreading group, suggesting that these patients presented a severe disease in their first medical evaluation. However, we found that a higher Tsui in the first medical evaluation (Tsui score $>7.5$ ) is not a predictor of progression, although it has a good association with the spreading outcome (likelihood ratio=3.81, $p=0.02$ ). We calculated our model for different subsets of candidate variables, but only those presented in Table 4 were significant. We did not expect that Tsui score $>7.5$ was not a good predictor. However, Tsui score depends of different variables (rotation, anterocollis, retrocollis, laterocollis, duration of sustained movements, shoulder elevation, and head tremor) and was not designed as prognostic score, but as follow-up one for the treatment with BTX-A ${ }^{9}$. We believe that Tsui score was ranked as candidate variable but did not become a reliable predictor, because it is influenced by the time of disease and other variables included in our model such as anterocollis, retrocollis and head tremor.

It is known that in patients with early-onset dystonia (age $<28$ years) the symptoms tend spread more than in those with late-onset dystonia ${ }^{1,10}$. Our patients presented a late-onset CD and when we compared onset-age between groups, we observed that patients in the spreading group were older than those in the non-spreading group (45.7 \pm 3.6 years vs. $37.1 \pm 1.9, p=0.02)$. In our model of logistic regression, we considered onset-age $<28$ years as a candidate variable, but it had a weak association with progression in our group of patients (likelihood ratio=0.09, $p=0.7$ ).

We also observed that patients with time of disease $>18.5$ months have a higher risk of spreading (risk ratio=2.4). In our group of patients, those who spreaded their disease, presented this event in $24\left(12-38,25^{\text {th }}\right.$ and $75^{\text {th }}$ percentiles) months. Our data are in line with a previous report ${ }^{3}$, which concluded that most patients with $C D$ deteriorate during the first five years. Spontaneous remission of CD is also described ${ }^{4,11}$, but we did not observe it in any of our patients. However, we must report that our patients in the non-spreading group are younger and present similar period of follow-up, when compared to the spreading one. We do not know if these patients, in a longer follow-up, will also spread, once they present the predictors of the spreading group: time of disease $>18$ months, anterocollis, retrocollis and head tremor.

The pattern of CD spread in our group of patients was noteworthy and somewhat different from previous reports that showed a predilection for spread to the cranial segment or to the upper limb $b^{6,712}$. In fact, most of patients spreaded to cranial segment, but we found a high prevalence of generalized spreading ( $28 \%$ of patients). Unfortunately, we did not test them for DYT7 mutation; it is known that patients with CD who are DYT7 mutation carriers are more prone to spread to generalized dystonia, ${ }^{1,13}$.

Our study presented some limitations, especially due to its retrospective nature. Although data were based on information obtained from medical charts with the participation of different physicians throughout the period of this study, the final diagnosis and Tsui scale score were always made concurrent at least with one of the authors (HBF, VB, SMAS, PMCA), and the same standard protocol was used for diagnostic purposes and follow-up. An information that we could not analyze was the influence of the treatment with BTX-A on spreading pattern. All patients received BTX-A (either Botox ${ }^{\circledR}$ or Dysport ${ }^{\circledR}$ ), but in different doses and number of injections.

In conclusion, our retrospective study showed that time of disease $>18.5$ months, retrocollis, anterocollis and head tremor are good clinical correlates to predict spreading of movement to other regions of the body of $C D$ patients. We think that further prospective studies with a long follow-up should address the time of CD spreading beyond the neck region and the influence of BTX-A on spreading.

\section{REFERENCES}

1. Bressman SB, Leon D, Raymond D, et al. Clinical-genetic spectrum of primary dystonia. Adv Neurol 1998;78:79-91.

2. de Carvalho Aguiar PM, Ozelius LJ. Classification and genetics of dystonia. Lancet Neurol 2002;1:316-325. 
3. Meares R. Natural history of spasmodic torticollis and effect of surgery. Lancet 1971;2:149-150.

4. Lowenstein DH, Aminoff MJ. The clinical course of spasmodic torticollis. Neurology 1988;38:530-532.

5. Jahanshahi M, Marion MH, Marsden CD. Natural history of adult-onset idiopathic torticollis. Arch Neurol 1990;47:548-552.

6. Weiss EM, Hershey T, Karimi M, et al. Relative risk of spread of symptoms among the focal onset primary dystonia. Mov Disord 2006;21:1175-1181.

7. Svetel M, Pekmezovic T, Jovic J, et al. Spread of primary dystonia in relation to initially affected region. J Neurol 2007;254: 879-883.

8. Camargo CH, Teive HA, Becker N, Baran MH, Scola RH, Wer- neck LC. Cervical dystonia: clinical and therapeutic features in 85 patients. Arq Neuropsiquiatr 2008;66:15-21.

9. Tsui JK, Eisen A, Stoessl AJ, et al. Double-blind study of botulinum toxin in spasmodic torticollis. Lancet 1986;2:245-247.

10. Koukouni V, Martino D, Arabia G, Quinn NP, Bhatia KP. The entity of young onset primary cervical dystonia. Mov Disord 2007;22:843-847.

11. Friedman A, Fahn S. Spontaneous remissions in spasmodic torticollis. Neurology 1986;36:398-400.

12. Andrade LAF, Ferraz HB. Idiopathic dystonia: clinical profile of 76 Brazilian patients. Arq Neuropsiquiatr 1992;50:426-432.

13. Chan J, Brin MF, Fahn S. Idiopathic cervical dystonia: clinical characteristics. Mov Disord 1991;6:119-126. 